\title{
Development of Intelligent Traffic Light System Based On Congestion Estimation Using Fuzzy Logic
}

\author{
Javed Alam ${ }^{1}$, Prof. (Dr.) M. K. Pandey ${ }^{2}$ \\ ${ }^{l}$ Research Scholar, Deptt of CS, Mewar University Gangrar, Chittorgarh, Rajasthan (INDIA) \\ ${ }^{2}$ Director Computer Science \& Applications, AIMCA, Haldwani UK (INDIA)
}

\begin{abstract}
Vehicular traffic is the major problem which every country faces because of the increase in number of vehicles throughout the world, especially in large urban areas. In a conventional traffic light controller, the traffic lights change at fixed time. It does not provide an optimal solution. Many traffic light controllers implemented in current practice, are based on the 'time-of-the-day' scheme, which uses a limited number of predetermined traffic light patterns and implement these patterns depending upon the time of the day. These automated systems do not provide an optimal control for fluctuating traffic volumes. A traffic light controller based on fuzzy logic can be used to optimize the control of unprecedented traffic volumes such as over saturated or unusual load conditions. Therefore, it is necessary to improve the traffic controller for effective traffic management and better traffic flow. The efficiency of traffic flow through an intersection depends on the phases, sequence and the timing of the traffic signals installed to minimize the wait time of the vehicle in each queue. Fuzzy optimization deals with finding the values of input parameters of a complex simulated system which results in desired output. Fuzzy logic controller is used to execute fuzzy logic inference rules from a fuzzy rule base in determining the congestion parameters, getting the warning information and the appropriate action. This paper, describe the design and implementation of an intelligent traffic light system (ITLS) based on congestion estimation using fuzzy logic. In an intelligent traffic light system (ITLS) number of vehicles in each lane is measured using sensors. At the end of each phase these numbers are used as inputs to fuzzy controller. Fuzzy controller calculates the duration of green light as per the traffic situation. To simulate the situation of an isolated traffic junction based on congestion estimation, we use MATLAB. The results obtained show an improvement in the overall outcome of traffic management as compared to the conventional traffic controller, marking great feasibility and practicality of the current model.
\end{abstract}

Keyword: Simulation, Fuzzy logic, ITLS, Arrival, Queue, Right-Queue, and Extension time.

\section{Introduction}

In India, every year nearly 85,000 persons are reported to be killed and 300,000 are injured due to accidents on road. An accident takes place every 3 minutes and a person killed every 10 minutes on Indian roads and this number is continuously increasing. Increase in accidents is coincident with the increase in vehicle population. More than ten million road accidents have taken place on Indian roads after motor vehicles came to India and almost 1.5 million people have lost their lives and many more have suffered injuries. The Indian share in world vehicle population is as small as $1 \%$ but its share of road accident is $6 \%$, while the motorization level is second lowest of the world [14]. Government is taking appropriate steps to improve the situation but the results are not encouraging. Every year numerous seminars are arranged on Road Safety. Road Safety Week is observed all over the country, yet the problem is increasing day by day. So to minimize the road accident events and for efficient vehicular movement another aspect to be considered is effective monitoring and control of traffic.

The monitoring and control of Road traffic is becoming a major problem in many countries. The increasing number of vehicles and the lower phase of highways developments have led to traffic congestion problem. There are many factors that lead to traffic congestion such as the density of vehicles on the roads, human habits, social behavior, and traffic light system. One major factor is due to the traffic light system that controls the traffic at junction. Traffic policeman are deployed at traffic intersection everyday in order to overcome these congestion during peak hour, thus one of the roots of the problem is due to ineffective traffic light controllers. With effective traffic control at the intersection, it is believed that the overall capacity and performance of urban traffic network could be resolve. With the ever increasing number of vehicles on the road, the Monitoring authorities have to find new ways or measures of overcoming such a problem. Many solutions were proposed to solve the traffic jam. Most conventional traffic surveillance systems use intrusive sensors, including inductive loop detectors, micro-loop probes, and pneumatic road tubes. However, these sensors disrupt traffic during installation and repair, which leads to a high cost installation and maintenance. In addition, over the ground sensors like videos, radars, and ultrasonic were used. These systems are also high cost and their accuracy depends on environment condition [1,2]. 
Conventional methods for traffic signal control based precise models fail to deal efficiently with the complex and varying traffic situations. These models are based on the preset cycle time to change the signal status without any analysis of traffic situation. Due to fixed cycle time, such systems do not consider that which intersection has more load of traffic, so should kept green more or should terminate earlier than complete cycle time. In case of intersections, conventional control systems only consider waiting time of signals on different directions but not the vehicle directions. Such situations can be seen in various areas of Dehradun, India like Darshanlal-chowk, Prince-chowk etc. where traffic flow varies in different hours and heavy traffic flows at a particular route in the morning and evening timings because of large number of offices on that route. Also, in different intersections, traffic flow abruptly changes in schools timings than non-peak hours. Preset Cycle Time Controllers fail in such scenarios because they could not get complete information of vehicles present in the queue earlier. Also, sometimes situation arises, when some VIP movement is there, the traffic flow has to be diverted to other available intersections. In such situations, efficiency of human decision-making is unprecedented because decision making objectives are unclear [1,2]. Fuzzy based controllers are proved to be well manager of traffic system in such scenarios. Fuzzy controllers have the ability to take decision even with incomplete information. More and more sophisticated controllers are being developed for traffic control [1, 5, 6 and 7]. These algorithms are continually improving the safety and efficiency by reducing the waiting delay of vehicles on signals. This increases the tempo of travel and thus makes signals more effective and traffic flow smooth. The key motivation towards Fuzzy Logic in traffic signal control is the existence of uncertainties in signal control. Decisions are taken based on imprecise information and the effect of evaluation is not well known [8].

In this paper we discuss the implementation of an intelligent traffic light system for isolated $\mathrm{T}$ junction using fuzzy logic based technology which has the capability of mimicking human intelligence for controlling traffic light. We used fuzzy logic tools available with MATLAB and developed software to simulate the situation of traffic at an isolated junction. The simulated model used for the analysis of efficiency of traffic light controller based on various traffic characteristics such as waiting time, density, cost etc. The software can also be used as an exercise for undergraduate and graduate students to understand the concept of fuzzy logic and its application to a real life environment. The rules and membership functions of the fuzzy logic controller can be selected and changed; their outputs can be compared in terms of several different representations. Fuzzy logic based technology allows the implementation of real-life rules similar to the way humans would think. In Traffic Control System, humans would think in the following way to control traffic situation at a certain junction: "if the traffic is heavier on the north or south lanes and the traffic on the west or east lanes is less, then the traffic lights should stay green longer for the north and south lanes". Such rules can now be easily accommodated in the fuzzy logic controller. In this consideration, we can say that it is replaceable to Traffic Police Officers. Fuzzy Logic works glowing when traffic flow in different directions is highly uneven as compared to Pretimed Controller. The beauty of fuzzy logic is that it allows vague terms and conditions such as "small", "Medium", and "large" to be quantized and understood by the computer [9].

\section{Literature Review}

In this section, we discuss different research work in the field of traffic light system. In other words this section concentrates on the use of fuzzy logic for traffic control. The first attempt made to design Fuzzy Traffic Controller was in 70s by Pappis and Mamdani [4]. After that Niittymaki, Kikuchi, Chui and other researchers [5] developed different algorithms and logic controllers to normalize traffic flow. Kelsey and Bisset also designed a simulator for signal controlling of an isolated intersection with one lane. Same work was also done by Niittymaki and Pursula [6]. They observed that Fuzzy Controller reduces the vehicle delay when traffic volume was heavy. Niittymaki and Kikuchi developed Fuzzy based algorithm for pedestrians, crossing the road.

Nakatsuyama, Nagahashi, and Nishizuka [7] applied fuzzy logic to control two adjacent intersections on an arterial with one-way movements. Fuzzy control rules were developed to determine whether to extend or terminate the green signal for the downstream intersection based on the upstream traffic. Chui was the first who uses Fuzzy Logic to control traffic in multiple intersections [5]. In this attempt, only two way streets are evaluated without considering any turnings.

Shruthi K R and Vinodha K [13]discuss a paper entitled "Priority based traffic lights controller using wireless sensor networks" In this research, two junctions are discussed and brought into focus along with the use of wireless sensors as many studies suggest the use of sensors. If there is no traffic lights control system in these junctions, huge amount of traffic causes waiting time and accidents. As a result, physical existence of traffic police is always required there which is inappropriate due to availability of technology today. Also, due to heavy traffic in these two junctions, emergency vehicles face hardships when they pass from there. Sensors will be located at a specific distance before the junctions which will detect the speed and sound waves of siren at a particular threshold. Based on the speed, sensors will communicate wirelessly with the traffic control system of the two junctions while realizing their routes. 
Bilal Ahmed Khan and Nai Shyan Lai [12] discuss a paper entitled "An intelligent traffic controller based on fuzzy logic". In this project two traffic signals are set in a T-junction configuration. Intelligence governed by the fuzzy logic controller has been linked to image processing which acts as a superior mode of data acquisition. The final stage comprises role of a microcontroller which recognizes and drives the traffic signals accordingly.

In recent years, Lin Zhang and Honglong Li [8] also worked on designing Fuzzy Traffic Controller for Oversaturated intersections. Jee-Hyong Lee and Hyung Lee-Kwang [9] presented direction-varying traffic signal control but assume that right turn traffic flow do not disturb any other traffic flows in an intersection.

\section{Fuzzy Logic}

The Fuzzy Logic tool was introduced in 1965, by Lotfali Askar Zadeh, better known as Lotfi A. Zadeh, is a mathematician, electrical engineer, computer scientist, artificial intelligence researcher and professor emeritus of computer science at the University of California, Berkeley. Fuzzy logic is a mathematical tool for dealing with uncertainty. It offers to a soft computing partnership, the important concept of computing with words. It provides a technique to deal with imprecision and information granularity. The fuzzy theory provides a mechanism for representing linguistic constructs such as "many," "low," "medium," "often," "few." In general, the fuzzy logic provides an inference structure that enables appropriate human reasoning capabilities. On the contrary, the traditional binary set theory describes crisp events, events that either do or do not occur. It uses probability theory to explain if an event will occur, measuring the chance with which a given event is expected to occur. The theory of fuzzy logic is based upon the notion of relative graded membership and so are the functions of mentation and cognitive processes. The utility of fuzzy sets lies in their ability to model uncertain or ambiguous data. Applications of fuzzy logic occur in three primary categories: consumer products, industrial/commercial systems and decision support systems [1,2].

\section{Mathematical Concept For Fuzzy Logic}

A fuzzy set is defined as the extension of a crisp (classical) set which allows only full membership or no membership to its elements (Zadeh, 1965). In a classical set $A$ the membership function define as-

$$
\mu_{A}(x)=\left\{\begin{array}{lll}
1, & (\text { True }) & x \in A \\
0, & (\text { False }) & x \notin A
\end{array}\right\}
$$

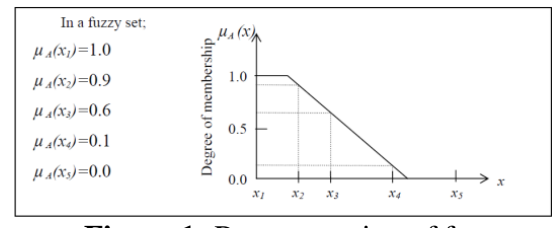

Figure 1: Representation of fuzzy set

Fuzzy set theory extends this concept by defining partial membership. A fuzzy set $A$ on a universe of discourse $U$ is characterized by a membership $\mu_{A}$ that takes values in the interval $[0,1]$. The membership functions, both linear and non-linear, that are most commonly used in engineering can be classified into four types as follows:

a.

Zadeh's $S: x \rightarrow[0,1]$ defined as

$$
S(x, \alpha, \sigma, \beta)=\left\{\begin{array}{l}
0 \\
2\left(\frac{x-\alpha}{\beta-\alpha}\right)^{2} \\
1-2\left(\frac{x-\beta}{\beta-\alpha}\right)^{2} \\
1
\end{array}\right.
$$

$$
\left.\begin{array}{ll}
\text { for } & x \leq \alpha \\
\text { for } & \alpha<x \leq \sigma \\
\text { for } & \sigma<x \leq \beta \\
\text { for } & x>\beta
\end{array}\right\}
$$

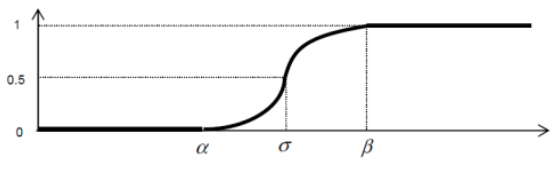

Figure 2: An example of S-function

b. The function $\mathrm{G}: \mathrm{x} \rightarrow[0,1]$ defined as

$$
\Gamma(x, \alpha, \beta)=\left\{\begin{array}{l}
0 \\
\left(\frac{x-\alpha}{\beta-\alpha}\right) \\
1
\end{array}\right.
$$

$$
\left.\begin{array}{ll}
\text { for } & x<\alpha \\
\text { for } & \alpha \leq x \leq \beta \\
\text { for } & x>\beta
\end{array}\right\}
$$

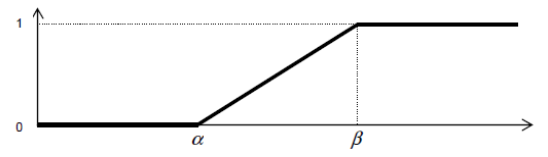

Figure 3: An example of $\Gamma$-function

c. The function $\mathrm{L}: \mathrm{x} \rightarrow[0,1]$ defined as 


$$
L(x, \alpha, \beta)=\left\{\begin{array}{l}
1 \\
\left(\frac{\beta-x}{\beta-\alpha}\right)
\end{array}\right.
$$

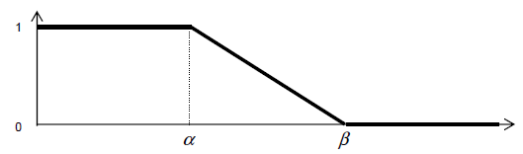

Figure 4: An example of L-function

d. The function $\mathrm{A}: \mathrm{x} \rightarrow[0,1]$ defined as

$$
A(x, \alpha, \sigma, \beta)=\left\{\begin{array}{l}
0 \\
\left(\frac{x-\alpha}{\sigma-\alpha}\right) \\
\left(\frac{\beta-x}{\beta-\sigma}\right) \\
0
\end{array}\right.
$$

$$
\left.\begin{array}{l}
\text { for } \quad x<\alpha \\
\text { for } \quad \alpha \leq x \leq \sigma \\
\text { for } \sigma<x \leq \beta \\
\text { for } x>\beta
\end{array}\right\}
$$

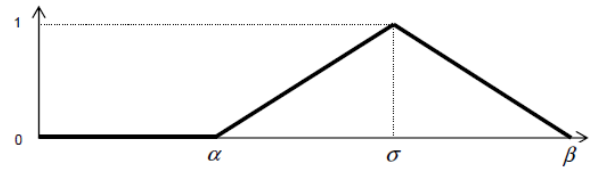

Figure 5: An example of A-function

\section{Description Of The Proposed Intelligent Traffic Light System (ITLS)}

The first traffic management systems used in Germany was implemented on roads with frequent accidents caused by fog or icy road conditions. Later, these systems were extended to detect and control traffic to increase the traffic capacity. These traffic control systems use several detection stations along the road. These stations employ magnetic sensors for traffic detection, as well as weather stations transmitting environmental data from road surface and the air layer near the ground. A central traffic control computer collects the data transmitted from the section stations. A control strategy derives an adequate speed limit for every section. The control objectives are:

- Keep traffic flowing in case of peak traffic

- Slow down traffic at the inflow to congestion

- Warn for bad weather conditions such as fog or ice

Along the road of such an "intelligent" highway, alterable road signs posted on traffic sign gantries display speed limits for each lane and display non regular events such as road work, warnings for traffic backups, breakdowns, an accident, or dangerous weather conditions [1,2]. Fuzzy logic traffic light control is an alternative to conventional traffic light control which can be used for a wider array of traffic patterns at an intersection. A fuzzy logic controlled traffic light uses sensors that count vehicles instead of proximity sensors which only indicate the presence of vehicles. This provides the controller with traffic densities in the lanes and allows a better assessment of changing traffic patterns. As the traffic distributions fluctuate, the fuzzy controller can change the signal light state accordingly.

The general structure of a fuzzy intelligent traffic light system is illustrated as in Figure-6. There are two electromagnetic sensors placed on the road for each lane at $\mathrm{T}$ junction. The first sensor behind each traffic light counts the number of cars passing the traffic light, and the second sensor which is located behind the first sensor counts the number of cars coming to the intersection at distance $S$ from the light. The number of cars at traffic light is determined by the difference of the reading between the two sensors. This is in contrast to conventional control systems which place a proximity sensor at the front of each traffic light and can only sense the presence of a car waiting at the junction. The distance between the two sensors $\mathrm{S}$, is determined accordingly following the traffic flow pattern at that particular intersection. The fuzzy logic controller is responsible for controlling the length of the green time according to the traffic conditions. The state machine controls the sequence of states that the fuzzy traffic controller should cycle through. There is one state for each phase of the traffic light. There is one default state which takes place when no incoming traffic is detected. This default state corresponds to the green time for a specific approach, usually to the main approach. In the sequence of states, a state can be skipped if there is no vehicle queues for the corresponding approach.

In this research, the main goals of fuzzy logic in the traffic signal control, and a matter of fact, also in traffic signal control in general, are as follows-

- Improving of traffic safety in the Tjunction.

- Save the time of human being.

- Maximizing the capacity of the intersection.

- Minimizing the delays.

- Clarifying the traffic environment.

- Influencing the route choices.

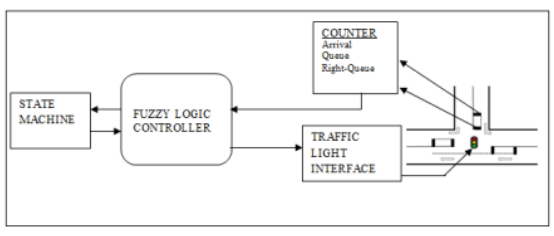

Figure 6: General Structure of intelligent fuzzy system 


\section{Methodology}

In the development of intelligent traffic light system the following assumptions are made:

- The junction is an isolated T-junction or 3 way intersection with traffic coming from all the sides as shown in figure 7 and figure 9.

- Left movement of each lane is always free.

- When traffic moves from one direction other two directions traffic will remain stop.

- The fuzzy logic controller will observe the density of all the sides.

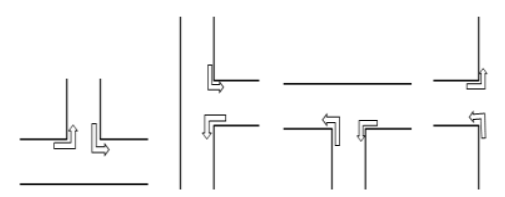

Figure 7: Basic Structure of T-junction with left turn free

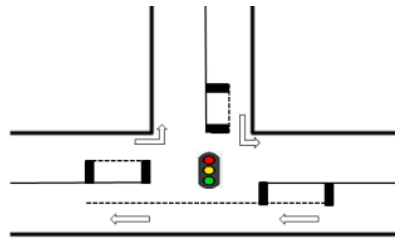

Figure 8: Special structure of Tjunction

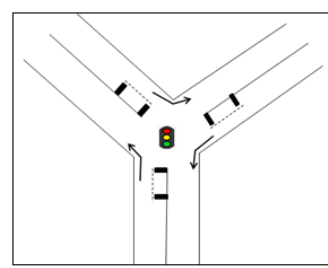

Figure 9: General Structure of 3-way intersection with left turn free

The flow diagram of a fuzzy logic controller is shown in figure 10. A fuzzy logic controller was designed for an isolated T-junction or 3 way intersection as shown in figure 8 and figure 9 . In the traffic light controller three fuzzy input variables are chosen: number of vehicles on arrival side (Arrival), number of vehicles on queuing side (Queue), number of vehicles on right side (Right-Queue).

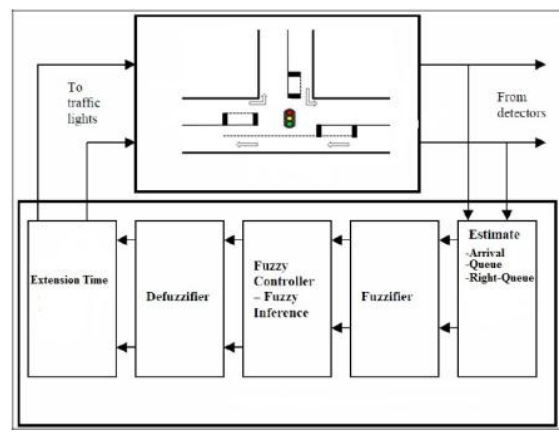

Figure 10: Fuzzy logic Controller for Traffic Signal

In an intelligent traffic light system traffic light work in clock wise or anti-clock wise direction. If the one side is green then this would be the Arrival side while second side would be considered as Queue and third side as Right-Queue. The output fuzzy variable (Extension) would be the extension time needed for the green light on the Arrival. Thus based on the current traffic conditions the fuzzy rules can be formulated so that the output of the fuzzy controller will extend or not the current green light time. If there is no extension of the current green time, the state of the traffic light will immediately change to another state, allowing the traffic from the alternate phase to flow.

\section{Fuzzy Parameters And Their Membership Functions Design}

For the traffic light control, there are four membership functions for each of the input as well as output fuzzy variable of the system. Table 1, shows the fuzzy input variables of Arrival, Queue, and Right-Queue and output variable Extension time of the system.

Table 1

Fuzzy Variables form for Intelligent traffic light system

\begin{tabular}{|c|c|c|c|c|c|}
\hline \multicolumn{3}{|c|}{ Input Variables } & \multirow{2}{*}{ Abbreviations } & \multirow{2}{*}{\multicolumn{2}{|c|}{$\begin{array}{c}\text { Output Variable } \\
\text { Extension }\end{array}$}} \\
\hline Queue & Arrival & Right-Queue & & & \\
\hline Small & \begin{tabular}{|l|} 
Small \\
\end{tabular} & Small & $\mathrm{s}$ & & $\mathrm{Z}$ \\
\hline Medium & Medium & Medium & M & Small & S \\
\hline Large & Large & Large & $\mathrm{L}$ & Medium & M \\
\hline Very-Large & Very-Large & Very-Large & $\mathrm{VL}$ & Large & $\mathrm{L}$ \\
\hline
\end{tabular}

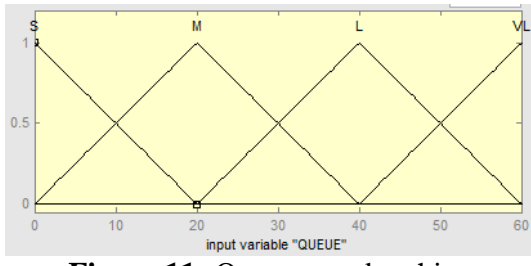

Figure 11: Queue membership

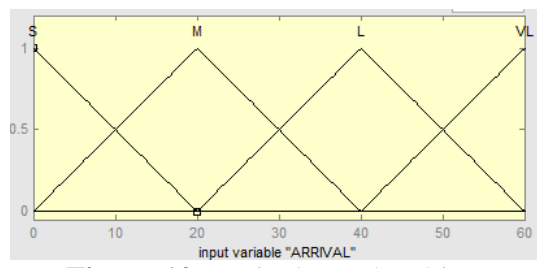

Figure 12: Arrival membership 


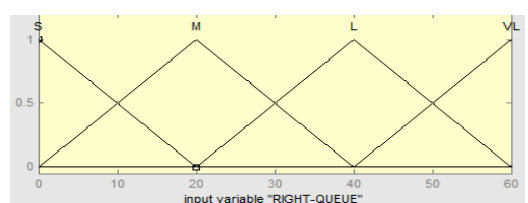

Figure 13: Right-Queue membership

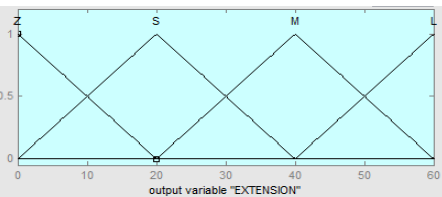

Figure 14: Extension time membership

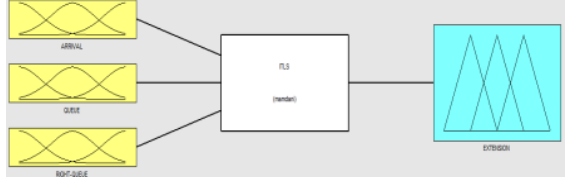

Figure 15: The whole design structure using Mamdani Method

\section{Fuzzy Rules}

The inference mechanism in the fuzzy logic controller resembles that of the human reasoning process. This is where fuzzy logic technology is associated with artificial intelligence. Humans unconsciously use rules in implementing their actions. For example, a traffic policeman manning a junction say, one from the north and one from the west; he would use his expert opinion in controlling the traffic more or less in the following way:

\section{IF traffic from the one side of the city is HEAVY AND traffic from the other sides is LESS THEN allow movement of traffic from the one side LONGER}

In Intelligent traffic light system 64 rules have been found. The some fuzzy rule uses in intelligent traffic light system shown in the figure 16.

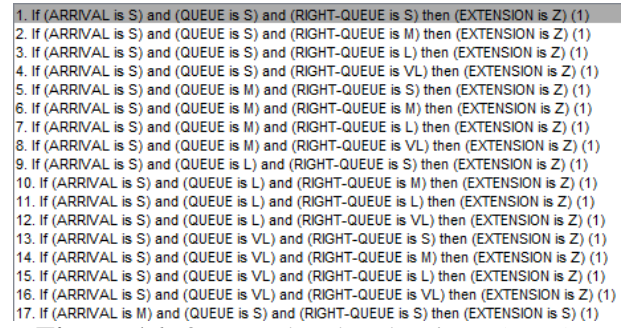

Figure 16: fuzzy rules develop in MATLAB

\section{Inference Engine And Defuzzification}

The basic function of the inference engine is to compute level of belief in output fuzzy sets from the levels of belief in the input fuzzy sets. The output is a single belief value for each output fuzzy set. In this stage, the fuzzy operator is applied in order to gain a single number that represents the result of the antecedent for that rule. The procedure of converting each aggregated fuzzy output set into a single crisp value is called defuzzification. In an intelligent traffic light system (ITLS), we use the following defuzzification method-

- Centroid method $(\mathrm{CoA} / \mathrm{CoG})$

- Bisector Method

- Smallest of Maximum (SOM)

- Mean of Maximum (MOM)

- $\quad$ Largest of Maximum (LOM)

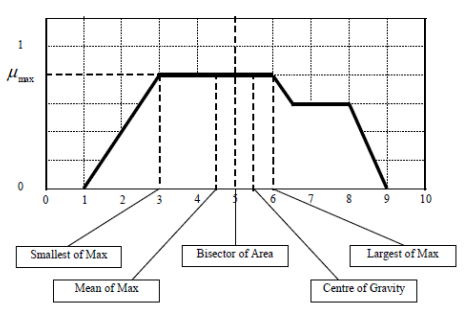

Figure 17: Defuzzification method

In the fuzzy logic controller once the appropriate rules are fired, the degree of membership of the output fuzzy variable i.e., Extension time, is determined by encoding the antecedent fuzzy subsets, in this case Queue, Arrival, and Right-Queue. In Intelligent traffic light system using fuzzy control, the max-min implication technique is used. Using this technique, the final output membership function for each rule is the fuzzy set assigned to that output by clipping the degree of truth values of the membership functions of the associated antecedents. Once the membership degree of each output fuzzy variable is determined, all of the rules that are being fired are then combined and the actual crisp output is obtained through defuzzification.

\section{Simulation Result And Discussion}

After the Intelligent traffic light system was carefully designed, we test the system and discuss the impact of the input variables on the output variable. With the help of simulation we show the effect of the three inputs to resulted extension time. This system shows the slow growing in the time that will added to the cycle which make the traffic situation more stable. 
As shown in Figure 18, the extension time (z-axis) using centroid method is small when the density of arrival side (x-axis) is small and the density of the queue side (y-axis) also small. Unlike the other defuzzification method, here the extension time grows slowly its being large only when the arrival side density is very close to large values and the queue side density is very close small value. This is important point of difference among methods.

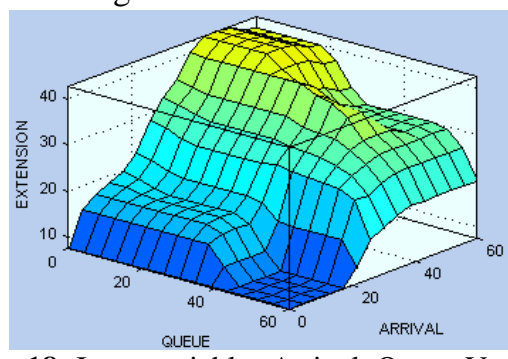

Figure 18: Input variables Arrival, Queue Vs output variable Extension

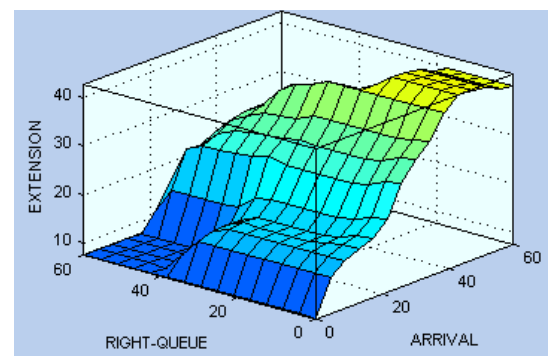

Figure 19: Input variables Arrival, Right-Queue Vs output variable Extension

The Figure 19 have similar parts of Figure 18, the extension time (z-axis) is small when the arrival side (x-axis) density have almost to many value and the right queue (y-axis) have a value from medium to long. The extension time grow fastly and gets a maximum value when the arrival side is being too many and the right turn density become very small.

As shown in Figure 20, the extension time is close to zero when both the queue side density and the right queue are between medium to long values. Then the extension time grow slowly and have a long value when both the queue side and the right queue are very small. Also in this case we observe that the centroid defuzzification method will reduce the time as much as possible.

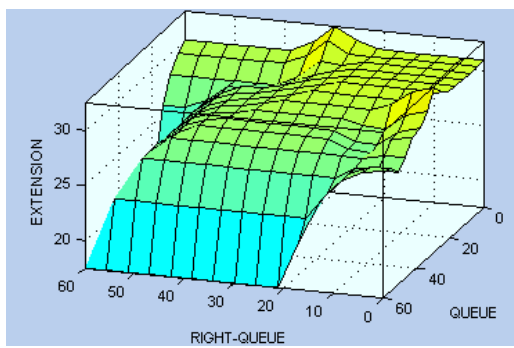

Figure 20: Input variables Queue, Right-Queue Vs output variable Extension

The simulation implemented in five stages as Table 2. In each stage we test one of the inputs with other inputs and discuss their effect on the output extension time with different defuzzification method.

Table 2

Extension time by various defuzzification methods at different values of input variables Arrival, Queue, Right-Queue side

\begin{tabular}{|c|c|c|c|c|c|c|c|}
\hline \multicolumn{3}{|c|}{$\begin{array}{c}\text { No. of Vehicles at } \\
\text { (Max Range=60) }\end{array}$} & \multicolumn{5}{|c|}{$\begin{array}{c}\text { Extension time } \\
\text { By Mamdani Method } \\
\text { (Max Range=60) }\end{array}$} \\
\hline $\begin{array}{c}\text { Arrival } \\
\text { side }\end{array}$ & $\begin{array}{c}\text { Queue } \\
\text { side }\end{array}$ & $\begin{array}{c}\text { Right-Queue } \\
\text { side }\end{array}$ & CENTROID & BISECTOR & MOM & LOM & SOM \\
\hline 5 & 5 & 5 & 13.9 & 10.2 & 2.4 & 4.8 & 0 \\
\hline 10 & 10 & 10 & 17.5 & 17.4 & 15 & 30 & 0 \\
\hline 20 & 20 & 20 & 20 & 19.8 & 19.8 & 19.8 & 19.8 \\
\hline 30 & 30 & 30 & 30 & 30 & 30 & 60 & 0 \\
\hline 40 & 40 & 40 & 40 & 40.2 & 40.2 & 40.2 & 40.2 \\
\hline 50 & 50 & 50 & 30 & 30 & 30 & 49.8 & 10.2 \\
\hline 60 & 60 & 60 & 20 & 19.8 & 19.8 & 19.8 & 19.8 \\
\hline 20 & 5 & 5 & 20 & 19.8 & 19.8 & 24.6 & 15 \\
\hline 30 & 5 & 5 & 30.9 & 27.6 & 31.8 & 60 & 10.2 \\
\hline 40 & 5 & 5 & 53.2 & 54 & 57.6 & 60 & 55.2 \\
\hline 50 & 5 & 5 & 44.5 & 47.4 & 55.2 & 60 & 50.4 \\
\hline 60 & 5 & 5 & 46.1 & 49.8 & 57.6 & 60 & 55.2 \\
\hline 5 & 10 & 5 & 15.5 & 12.6 & 4.8 & 9.6 & 0 \\
\hline 5 & 20 & 5 & 13.9 & 10.2 & 2.4 & 4.8 & 0 \\
\hline 5 & 50 & 5 & 15.5 & 12.6 & 4.8 & 9.6 & 0 \\
\hline 5 & 60 & 5 & 6.83 & 6 & 2.4 & 4.8 & 0 \\
\hline 5 & 5 & 10 & 15.5 & 12.6 & 4.8 & 9.6 & 0 \\
\hline 5 & 5 & 20 & 13.9 & 10.2 & 2.4 & 4.8 & 0 \\
\hline 5 & 5 & 50 & 15.5 & 12.6 & 4.8 & 9.6 & 0 \\
\hline 5 & 5 & 60 & 6.83 & 10.2 & 2.4 & 4.8 & 0 \\
\hline 10 & 5 & 2 & 17.5 & 17.4 & 15 & 30 & 0 \\
\hline 20 & 10 & 5 & 20 & 19.8 & 20.1 & 30 & 10.2 \\
\hline 30 & 15 & 8 & 30.9 & 27.6 & 31.8 & 60 & 10.2 \\
\hline 40 & 20 & 10 & 52.4 & 52.8 & 55.2 & 60 & 50.4 \\
\hline 50 & 25 & 13 & 42.5 & 42.6 & 45 & 60 & 30 \\
\hline 60 & 30 & 15 & 40 & 40.2 & 39.9 & 49.8 & 30 \\
\hline
\end{tabular}

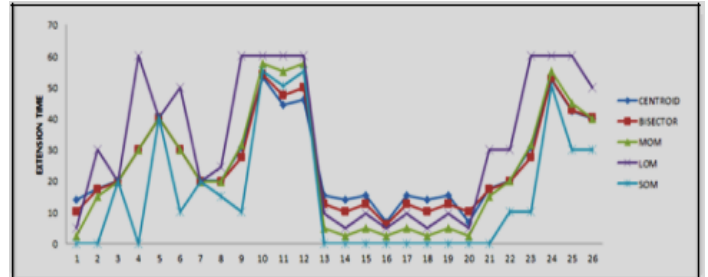

Figure 21: Extension time using different defuzzification method 
In figure 21 as well as in Table 2 compare different defuzzification methods used in Mamdani controller. The results of the methods are very close, but when we take a careful look to the methods we can observe that the result of centroid is less than the other methods of Mamdani.

As shown in Table 3 average of extension time by centroid method is less than largest of maxima (LOM) method. Although the result that produced from centroid defuzzification method are very close to other result. But standard deviation as shown in figure 22 and Table 3 is less than the other method also coefficient of variation has less value. Hence centroid defuzzification method provides less extension time as well as deviation than the other methods without making unstable conditions in the traffic flow.

For calculating average $(\overline{\mathrm{x}})$, standard deviation $(\sigma)$ and coefficient of variation $(\mathrm{cv})$, we use the following formula-

$$
\overline{\mathrm{x}}=\frac{\sum x}{n}, \quad \sigma=\sqrt{\frac{\sum(\mathrm{x}-\overline{\mathrm{x}})^{2}}{\mathrm{n}}}, \quad \mathrm{cv}=\frac{\sigma}{x} \times 100
$$

Where $\boldsymbol{x}$ is the extension time and $\mathbf{n}$ is the no. of observation, as shown in Table 2.

Table 3

Analysis of defuzzification method

\begin{tabular}{|c|c|c|c|c|c|}
\hline & CENTROI & BISECTOR & MOM & LOM & SOM \\
\hline Average & 25.88 & 25.13 & 23.65 & 32.17 & 15.65 \\
\hline $\begin{array}{c}\text { Standard } \\
\text { Deviation }\end{array}$ & 13.90 & 15.02 & 19.38 & 22.84 & 19.65 \\
\hline $\begin{array}{c}\text { Coefficient } \\
\text { of Variation }\end{array}$ & 53.71 & 59.78 & 81.94 & 71.00 & 125.54 \\
\hline
\end{tabular}

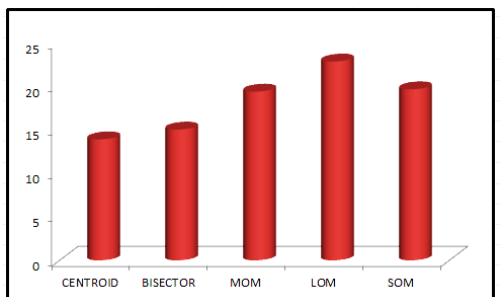

Figure 22: Standard deviation of different defuzzification method

As shown in figure 23 and Table 4 result of both the methods are very close. In maximum situation extension time taken by ITLS is similar to actual method. But ITLS take less time than fixed time controller without making unstable conditions in the traffic flow. Extension time of actual method are found with the help of the following formula-

$$
\text { Extension Time }=\frac{\text { Average Distance }}{\text { Average Speed }}
$$

Where average distance is 100 meter $(80+20)$ the distance from last vehicle in the queue to cover the intersection and average speed is $7.2 \mathrm{~km} / \mathrm{h}$ or $2 \mathrm{~m} / \mathrm{sec}$.

Table 4

Comparison of ITLS and actual method

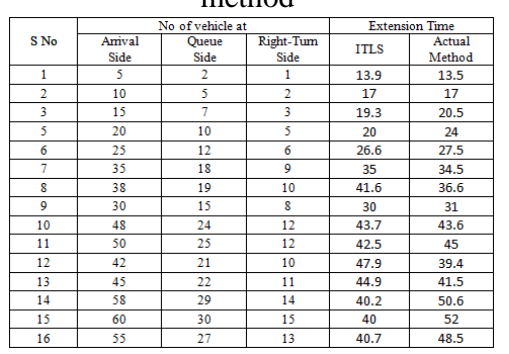

Table 5

Improvement results with different traffic condition

\begin{tabular}{|c|c|c|c|}
\hline \multirow{2}{*}{} & \multicolumn{3}{|c|}{$\begin{array}{c}\text { Average Extension Time } \\
\text { in Second }\end{array}$} \\
\cline { 2 - 4 } & $\mathbf{l}$ & $\mathbf{2}$ & $\mathbf{3}$ \\
\hline ITLS & 19.36 & 38.46 & 42.82 \\
\hline Actual Method & 20.5 & 38.14 & 46.4 \\
\hline Fixed Time System & 30 & 50 & 60 \\
\hline $\begin{array}{c}\text { Improvement } \\
\text { ITLS Vs. Actual Method }\end{array}$ & $5.56 \%$ & $-0.83 \%$ & $7.72 \%$ \\
\hline $\begin{array}{c}\text { Improvement } \\
\text { ITLS Vs. Fixed Time System }\end{array}$ & $35.47 \%$ & $23.08 \%$ & $28.63 \%$ \\
\hline
\end{tabular}

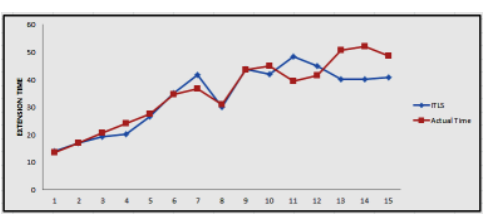

Figure 23: Analysis of ITLS and actual method

Table 5 shows the improvement percentage of ITLS with actual and fixed time system which is taken with different traffic condition. It can be observed from the results that intelligent traffic light system (ITLS) is superior to any classical or timing control methods. It provides better performance in terms of total waiting time as well as total moving time as compared to fixed time system.

\section{Conclusion And Future Works}

The fuzzy logic traffic light system performed better than the fixed time system or even vehicle actuated system due to its flexibility. The flexibility involves the number of vehicles sensed at the incoming junction and the extension of the green time. In the fixed time system, being an open loop system the green time is not extended whatever the density of car at the junction. In addition to the fuzzy variables as mentioned, the fuzzy system also has an advantage of performing according to linguistics rules in the manner of how a human 
would use.

In ITLS, extension time is not a fixed value. They are all fuzzy variables such as zero, small, medium and large. The numbers of vehicles sensed at the input of the fuzzy controller are also converted into fuzzy values, such as small, medium, large and very large. In addition to the fuzzy variables as mentioned, the fuzzy controller also has an advantage of performing according to linguistic rules in the manner of how a human behaves. The reasoning method in the fuzzy controller is also similar to that of the policeman handling the traffic flow at a typical junction.

It can be observed from the results that intelligent traffic light system provides better performance in terms of total waiting time as well as total moving time less waiting time will not only reduce the fuel consumption but also reduce air, noise pollution and save the time of human being. Overall, the simulation results indicated that ITLS has the potential to improve operations at T-junction. It also shows that it can reduce the traffic congestion and avoids the time being wasted by a green light on an empty road.

Some deeper problems need a further research. For example, it is assumed that arrival, queue and rightqueue lengths can be observed accurately, and there are no pedestrians. To apply the proposed ITLS method in the real world, it is necessary to conduct future studies that take pedestrians into account. More input variables like weather conditions and other environment conditions can be added to have more précised traffic control. Also VIP movement is not considered into proposed ITLS.

\section{References}

[1]. Javed Alam and Prof. (Dr.) M. K. Pandey “Advance Traffic Light System Based On Congestion Estimation Using Fuzzy Logic" in International Journal of Emerging Technology \& Advanced Engineering (IJETAE) ISSN 2250-2459, ISO 9001:2008, Volume 4, Issue 3, March 2014, PP 870-877.

[2]. Javed Alam, Prof. (Dr.) M.K. Pandey and Husain Ahmed "Intelligent Traffic Light Control System for Isolated Intersection Using Fuzzy Logic" International conference on Advances in Communication and Control Systems 2013 (CAC2S 2013) DIT University Dehradun, India, ISBN-978-90-78677-66-6, 6-8 ${ }^{\text {th }}$ April 2013, PP 209-215

[3]. Javed Alam and Prof. (Dr.) M.K. Pandey "Development of Traffic Light Control System for Emergency Vehicle Using Fuzzy Logic" International Conference on Artificial Intelligence and Soft Computing, IIT- BHU Varanasi, India 7-9 December-2012.

[4]. Pappis, C. P., and E. H. Mamdani. “A Fuzzy Logic Controller for a Traffic Junction”. IEEE Transactions on Systems, Man, and Cybernetics, Vol. SMC-7, No. 10, October 1977, pp. 707-717.

[5]. Chiu, S. "Adaptive Traffic Signal Control Using Fuzzy Logic”. Proceedings of the IEEE Intelligent Vehicles Symposium, 1992, pp. 98-107.

[6]. Niittymaki, J., and M. Pursula. "Signal Control Using Fuzzy Logic. Fuzzy Sets and Systems”, Vol. 116, 2000, pp. 11-22.

[7]. Nakatsuyama, M., H. Nagahashi, and N. Nishizuka. "Fuzzy Logic Phase Controller for Traffic Junctions in the One-Way Arterial Road". Proceedings of the IFAC Ninth Triennial World Congress, 1984, pp. 2865-2870.

[8]. Li, H., P. D. Prevedouros, and L. Zhang. "Signal Control for Oversaturated Intersections Using Fuzzy Logic" Submitted for consideration for presentation at the 2005 Annual Meeting of the TRB and publication in the Transportation Research Record.

[9]. Hong Wei, Wang Yong, Mu Xuanqin and Wu Yan. "A cooperative fuzzy control method for traffic Lights". 2001 IEEE Intelligent Transportation Systems Conference Proceedings - Oakland (CA), USA - August 25-29, 2001.

[10]. Yi Hu, CQU, Peter Thomas, Member, IEEE, and Russel J. Stonier, Member, IEEE. "Traffic Signal Control using Fuzzy Logic and Evolutionary Algorithms". 2007 - IEEE.

[11]. J Niittymaki, R Nevala, E Turunen. "Fuzzy Traffic Signal Control and a New Inference Method! Maximal Fuzzy Similarity - Fuzzy Sets and Systems", 2003.

[12]. Bilal Ahmed Khan and Nai Shyan Lai "An intelligent traffic controller based on fuzzy logic" SDIWC (ISBN: 978-0-9853483-8-0) 2013 PP 89-93.

[13]. Shruthi K R and Vinodha. K "Priority based traffic lights controller using wireless sensor networks" International Journal of Electronics Signals and Systems (IJESS) ISSN: 2231- 5969, Vol-1 Iss-4, 2012 PP 58-61

[14]. Pasricha, P.S.(1997), Road Accident Management Strategies, Indian Journal of Transport Management, Oct 1997, in Road SafetyA book of readings published Centre of Road Safety, Central Institute for Road Safety, Pune. 